\title{
Faddeev-Popov Ghost and BRST Symmetry in Yang-Mills Theory
}

\author{
Edyharto Yanuwar*, Jusak Sali Kosasih \\ Theoretical Physics Laboratory, Bandung Institute of Technology, \\ No. 10 Ganesha Street, Bandung, 40132, Indonesia
}

( Received: May 28, 2018, Revised: August 31, 2019, Accepted: February 11, 2020 )

\begin{abstract}
Ghost fields arise from the quantization of the gauge field with constraints (gauge fixing) through the path integral method. By substituting a form of identity, an effective propagator will be obtained from the gauge field with constraints and this is called the Faddeev-Popov method. The Grassmann odd properties of the ghost field cause the gauge transformation parameter to be Grassmann odd, so a BRST transformation is defined. Ghost field emergence with Grassmann odd properties can also be obtained through the least action principle with gauge transformation, and thus the relations between the BRST transformation parameters and the ghost field is obtained.
\end{abstract}

Keywords: BRST transformation, Faddeev-Popov method, gauge field, gauge transformation, ghost field, least action principle

\section{INTRODUCTION}

The gauge field Lagrangian with constraints is not necessarily gauge invariant, therefore one constructs an effective Lagrangian from the gauge field. While the path integral quantization of the Yang-Mills theory with constraints forces the effective Lagrangian to have a ghost term in it. The existence of the ghost term in Lagrangian effectively shows that the system phase-space has been extended with the ghost degree of freedom. From this ghost freedom one defines BRST transformation with the transformation parameter which is similar to a ghost field. However, the presence of the ghost term in effective Lagrangian through path integral quantization does not show explicit relations between the gauge transformation parameter and the ghost field.

In this paper, it will be shown that the ghost fields and gauge transformation parameters have relations by using the least action principle. In order to show the validity of these relations, we compare effective Lagrangian obtained from the least action principle, BRST variations and path integrals.

\footnotetext{
* Corresponding author.

E-mail address: edyyanuwar@gmail.com
}

The first part of this paper provides an overview of the gauge transformation. The second part explains the path integral quantization and the Faddeev-Popov method in finding effective propagators that leads to effective Lagrangian. While the third part discusses the least action principle which shows that the ghost field is a gauge transformation parameter with Grassmann odd properties. This property exists to make sure that the effective Lagrangian is gauge invariant. So that the BRST transformation is a gauge transformation with Grassmann odd parameters. Therefore, any Lagrangian that gauges invariant must be BRST invariant. This is explained in the fourth part about BRST symmetry.

In addition, the BRST operator is defined to be nilpotent which makes the second variation always zero. This property makes any Lagrangian with a BRST variation form to have an invariant BRST action. This helps in determining the effective Lagrangian.

\section{GAUGE TRANSFORMATION}

The gauge transformation of the gauge field can be expressed as $[1,2]$ 


$$
\begin{aligned}
& A_{\mu}^{\prime}=A_{\mu}-f_{b c}^{a} t_{a} A_{\mu}^{b} \alpha^{c}\left(x^{\mu}\right)+\frac{1}{g} t_{a} \partial_{\mu}\left(\alpha^{a}\left(x^{\mu}\right)\right) \\
& A_{\mu}^{a^{\prime}}=A_{\mu}^{a}-f_{b c}^{a} A_{\mu}^{b} \alpha^{c}\left(x^{\mu}\right)+\frac{1}{g} \partial_{\mu}\left(\alpha^{a}\left(x^{\mu}\right)\right)
\end{aligned}
$$

where $f_{b c}^{a}$ is the structure constant of the gauge group, $g$ is the gauge field coupling constant, $t_{a}$ is the gauge group generator, $\alpha^{c}\left(x^{\mu}\right)$ is the gauge transformation parameter that dependent on spacetime, and $a, b, c$ indexes expresses the internal symmetry index of the gauge field.

Lagrangian of Yang-Mills field with Lorenz gauge condition is

$$
\mathcal{L}=-\frac{1}{4} F_{\mu \nu}^{a} F_{a}^{\mu \nu}-\frac{1}{2 \xi} \sum_{a}\left(\partial_{\mu} A_{a}^{\mu}\right)\left(\partial^{v} A_{\nu}^{a}\right)
$$

where the Yang-Mills field Lagrangian is gauge invariant. Gauge fixing Lagrangian (Lorenz gauge) is not necessarily invariant so effective action and Lagrangian are needed to find the propagator of $A_{\mu}$ field as follows

$$
Z=\left\langle A_{\mu_{i}} t_{i} \mid A_{\mu_{f}} t_{f}\right\rangle=\int D A_{\mu} e^{i s}
$$

\section{FADDEEV-POPOV METHOD}

The Faddeev-Popov method substitutes identity into eq. (4) so that an effective propagator is obtained from the gauge field with constraints. The gauge invariant form of identity is given by $[2,3]$

$$
1=\Delta_{F}\left(A_{\mu}^{U}\right) \int D \alpha \delta\left(F^{a}\left[A_{\mu}^{U}\right]\right)=
$$

$\Delta_{F}\left(A_{\mu}\right) \int D \alpha \delta\left(F^{a}\left[A_{\mu}\right]\right)$.

The $\int D \alpha$ factor can be separated because the integrant does not depend on $\alpha$ so that the $Z$ propagator can be written as

$$
Z=\int D A_{\mu} \Delta_{\mathrm{F}}\left(A_{\mu}\right) \delta\left(F^{a}\left[A_{\mu}\right]\right) e^{i S} .
$$

Whilst, from the form of identity obtained [3]

$$
\Delta_{\mathrm{F}}\left(A_{\mu}\right)=\operatorname{det}\left|\frac{\delta F^{a}}{\delta \alpha}\right|_{F^{a}=0}=\operatorname{det} M
$$

with the integral representation of the determinant expressed in the Gaussian integral of the anticommuting field (Grassmann odd) [4]. In the case of finite dimensions, the determinant can be represented as

$$
\operatorname{det} M=\int D \bar{c} D c e^{i \int i \bar{c}^{a} M_{a b} c^{b} d^{4} x}
$$

where $\left(\bar{c}^{a}, c^{b}\right)$ is the anticommuting field (Grassmann odd) with properties

$$
\left.\begin{array}{c}
c^{i} c^{j}=-c^{j} c^{i}, \\
\bar{c}^{i} \bar{c}^{j}=-\bar{c}^{j} \bar{c}^{i}, \\
\bar{c}^{i} c^{j}+c^{j} \bar{c}^{i}=\delta^{i j} e
\end{array}\right\}
$$

The $c^{b}$ and $\bar{c}^{a}$ fields are independent Grassmann odd fields (both fields have different internal symmetries and Grassmann algebra, although both form Grassmann co-algebra). The $c^{b}$ field is usually called the ghost field and $\bar{c}^{a}$ is called the anti-ghost field.
General Lorenz gauge $F^{a}\left[A_{\mu}\right]=\partial^{\mu} A_{\mu}^{a}+$ $C^{a}$, where $C^{a}$ is an arbitrary function, gives $\delta\left(F^{a}\left[A_{\mu}\right]\right)=C^{a}$. The partition function $Z$ is not affected by $C^{a}$ because $\delta\left(F^{a}\left[A_{\mu}\right]\right)=C^{a}$ is a multiplier in propagator [2] so that the propagator will be given as

where

$$
Z=\int D A_{\mu} D \bar{c} D c e^{i \int \mathcal{L}_{\mathrm{eff}} d^{4} x}
$$

$$
\mathcal{L}_{\text {eff }}=\mathcal{L}_{Y M}+\mathcal{L}_{G F}+\mathcal{L}_{g h}=-\frac{1}{4} F_{\mu \nu}^{a} F_{a}^{\mu \nu}-
$$

$\frac{1}{2 \xi} \sum_{a}\left(\partial_{\mu} A_{a}^{\mu}\right)\left(\partial^{v} A_{v}^{a}\right)+i \overline{c^{a}} M_{a b} c^{b}$.

Now, $M_{a b}$ can be found through [1] with

$\operatorname{det} M=\operatorname{det}\left|\frac{\delta F^{a}\left[A_{\mu}\right]}{\delta \alpha}\right|_{F^{a}=0}=\operatorname{det}\left(-g \partial^{\mu} D_{\mu}^{a b}\right)$.

Therefore

$$
\mathcal{L}_{\text {eff }}=-\frac{1}{4} F_{\mu \nu}^{a} F_{a}^{\mu \nu}-\frac{1}{2 \xi} \sum_{a}\left(\partial_{\mu} A_{a}^{\mu}\right)\left(\partial^{v} A_{v}^{a}\right)-
$$

$i \bar{c}^{a} \partial^{\mu} D_{\mu}^{a b} c^{b}$.

For quantum electrodynamics with gauge group $U(1)$, the expression of the propagator $Z$ from the photon will be similar to equation (6), i.e.

$$
Z=\int D A_{\mu} \Delta_{\mathrm{F}}\left(A_{\mu}\right) \delta\left(F\left[A_{\mu}\right]\right) e^{i S}
$$

and the form of identity will give results similar to equation (7)

$$
\Delta_{\mathrm{F}}\left(A_{\mu}\right)=\operatorname{det}\left|\frac{\delta F}{\delta \alpha}\right|_{F=0}=\operatorname{det}\left(\frac{1}{e} \partial^{\mu} \partial_{\mu}\right) .
$$

By changing the determinant into an integral form, and because of ghost propagators do not affect electromagnetic propagators, electromagnetic propagators can be written separately from ghost propagators. Electromagnetic propagator is expressed by

where

$$
Z=\int D A_{\mu} e^{i \int \mathcal{L}_{Y M}+\mathcal{L}_{G F} d^{4} x},
$$

$$
\mathcal{L}_{Q E D}=-\frac{1}{4} F_{\mu \nu} F^{\mu \nu}-\frac{1}{2 \xi}\left(\partial_{\mu} A^{\mu}\right)\left(\partial^{v} A_{\nu}\right) .
$$

\section{LEAST ACTION AND GHOST FIELD}

After obtaining the ghost field through the Faddeev-Popov method, the ghost field will be constructed using the least action principle as performed in [5]. In this way explicit relations between the ghost fields and the transformation parameters are obtained.

We will review the consequences caused by adding gauge fixing Lagrangian (Lorenz gauge) to the Yang-Mills Lagrangian in equation (3). The action of a Lagrangian must be gauge invariant, so the action of $\mathcal{L}_{G F}$ must be gauge invariant. The proof is carried out through the least action principle on $\mathcal{L}_{G F}$ with gauge transformation as follows

$$
\int d^{4} x^{\prime} \mathcal{L}_{G F}^{\prime}=\int d^{4} x\left(\mathcal{L}_{G F}+\delta \mathcal{L}_{G F}\right),
$$


so it gives results

where

$$
\mathcal{L}_{G F} \rightarrow \mathcal{L}_{G F}^{\prime}=\mathcal{L}_{G F}+\delta \mathcal{L}_{G F}
$$

$$
\mathcal{L}_{G F}=-\frac{1}{2 \xi}\left(\partial_{\mu} A_{a}^{\mu}\right)\left(\partial^{v} A_{v}^{a}\right) .
$$

From equations (19) and (20) we obtaine

$\left.\partial_{v}\left(\alpha^{a}\left(x^{\mu}\right)\right)\right)$

so transformation (19) is not invariant. In order for transformation (19) to be invariant, take

$$
\begin{aligned}
& \partial^{v} D_{v}^{a b} \alpha^{b}\left(x^{\mu}\right)=\partial^{v}\left(g f_{b c}^{a} A_{\nu}^{b} \alpha^{c}\left(x^{\mu}\right)-\right. \\
& \left.\partial_{v}\left(\alpha^{a}\left(x^{\mu}\right)\right)\right)=0 .
\end{aligned}
$$

The parameter $\alpha^{a}$ in equation (22) can be considered as a field with the Lagrangian

$$
\mathcal{L}_{\alpha}=-i \bar{\alpha}^{a}\left(x^{\mu}\right) \partial^{v} D_{v}^{a b} \alpha^{b}\left(x^{\mu}\right)
$$

where $\bar{\alpha}^{a}\left(x^{\mu}\right)$ and $\alpha^{b}\left(x^{\mu}\right)$ are non-interrelated gauge transformation parameters.

From equations (3) and (23) the total Lagrangian is

$$
\mathcal{L}_{\text {tot }}=-\frac{1}{4} F_{\mu \nu}^{a} F_{a}^{\mu \nu}-
$$

$\frac{1}{2 \xi} \sum_{a}\left(\partial_{\mu} A_{a}^{\mu}\right)\left(\partial^{v} A_{v}^{a}\right)-i \bar{\alpha}^{a}\left(x^{\mu}\right) \partial^{v} D_{v}^{a b} \alpha^{b}\left(x^{\mu}\right)$,

so based on the least action principle

$$
\begin{aligned}
& \delta \mathcal{L}=\delta \mathcal{L}_{Y M}+\delta \mathcal{L}_{G F}+\delta \mathcal{L}_{\alpha}=0 \\
& \frac{1}{\xi g}\left(\partial_{\mu} A_{a}^{\mu}\right) \partial^{v} D_{v}^{a b} \alpha^{b}\left(x^{\mu}\right)+
\end{aligned}
$$

$i \bar{\alpha}^{a}\left(x^{\mu}\right) \partial^{v}\left(D^{\prime}{ }_{v}^{a b}-D_{v}^{a b}\right) \alpha^{b}\left(x^{\mu}\right)=0$.

The property of $\alpha^{b}\left(x^{\mu}\right)$ can be determined by taking $\delta \mathcal{L}_{\alpha}=0$ so that equation (22) is fulfilled, so that

$$
\begin{aligned}
& k \delta \mathcal{L}_{\alpha}=-g^{2} f_{c b}^{a} f_{e d}^{b} \partial^{v}\left(A_{v}^{d} \alpha^{e}\left(x^{\mu}\right) \alpha^{c}\left(x^{\mu}\right)\right)+ \\
& g f_{b c}^{a} \partial^{v}\left(\left(\partial_{v} \alpha^{b}\left(x^{\mu}\right)\right) \alpha^{c}\left(x^{\mu}\right)\right)
\end{aligned}
$$

where $k=i n \bar{\alpha}^{a^{\dagger}}, \quad n$ is the $\alpha^{b}\left(x^{\mu}\right)$ field normalization coefficient. Using

or

$$
f_{a b c} f_{c d e}=-\frac{1}{2} f_{j b d} f_{j a e}=\frac{1}{2} f_{j b d} f_{a j e}
$$

$$
f_{c b}^{a} f^{b}{ }_{e d}=-\frac{1}{2} f^{j}{ }_{c e} f_{j}^{a}{ }_{d}=\frac{1}{2} f^{j}{ }_{c e} f_{j d}^{a},
$$

then

$k \frac{\delta \mathcal{L}_{\alpha}}{g}=-\frac{1}{2} g f_{j d}^{a} \partial^{v}\left(A_{v}^{d}\left(f^{j}{ }_{c e} \alpha^{e}\left(x^{\mu}\right) \alpha^{c}\left(x^{\mu}\right)\right)\right)+$ $f_{b c}^{a} \partial^{v}\left(\left(\partial_{\nu} \alpha^{b}\left(x^{\mu}\right)\right) \alpha^{c}\left(x^{\mu}\right)\right)$.

The field $\left(f_{c e}^{a} \alpha^{e} \alpha^{c}\right)$ is substituted in the equation of motion of the field $\alpha^{a}\left(x^{\mu}\right)$ i.e. eq. (22), so that one obtains

$$
\begin{aligned}
& -g f_{j d}^{a} \partial^{v}\left(A_{v}^{d}\left(f_{c e}^{j} \alpha^{e} \alpha^{c}\right)\right)= \\
& \partial_{v} \partial^{v}\left(\delta_{j}^{a} f_{c e}^{j} \alpha^{e} \alpha^{c}\right) .
\end{aligned}
$$

Substituting equation (31) in equation (30) we get

$$
\begin{gathered}
k \frac{\delta \mathcal{L}_{\alpha}}{g}=-\frac{1}{2} f_{b c}^{a}\left(\partial^{v} \alpha^{b}\right)\left(\partial_{\nu} \alpha^{c}\right)+ \\
\frac{1}{2} f_{b c}^{a}\left(\partial_{\nu} \alpha^{b}\right)\left(\partial^{v} \alpha^{c}\right)-\frac{1}{2} f_{b c}^{a} \alpha^{b}\left(\partial^{v} \partial_{\nu} \alpha^{c}\right)+ \\
\frac{1}{2} f_{b c}^{a}\left(\partial^{v} \partial_{\nu} \alpha^{b}\right) \alpha^{c} .
\end{gathered}
$$

Equation (32) gives two possible relations between two parameter fields $\alpha^{b}$, which are Grassmann even or Grassmann odd.

For Grassmann even with commutation relations $\left[\alpha^{b}, \alpha^{c}\right]=0$, the $f_{b c}^{a}\left(\partial^{v} \alpha^{b}\right)\left(\partial_{v} \alpha^{c}\right)$ and $f_{b c}^{a}\left(\partial_{\nu} \alpha^{b}\right)\left(\partial^{v} \alpha^{c}\right)$ parts will satisfy

$$
f_{b c}^{a}\left(\partial^{v} \alpha^{b}\right)\left(\partial_{v} \alpha^{c}\right)=f_{b c}^{a}\left(\partial_{\nu} \alpha^{c}\right)\left(\partial^{v} \alpha^{b}\right)=
$$

$-f_{c b}^{a}\left(\partial_{v} \alpha^{c}\right)\left(\partial^{v} \alpha^{b}\right)=-f_{b c}^{a}\left(\partial_{v} \alpha^{b}\right)\left(\partial^{v} \alpha^{c}\right)$,

Likewise, the $f_{b c}^{a} \alpha^{b}\left(\partial^{v} \partial_{v} \alpha^{c}\right)$ and $f_{b c}^{a}\left(\partial^{v} \partial_{v} \alpha^{b}\right) \alpha^{c}$ parts will satisfy

$$
f_{b c}^{a} \alpha^{b}\left(\partial^{v} \partial_{v} \alpha^{c}\right)=f_{b c}^{a}\left(\partial^{v} \partial_{v} \alpha^{c}\right) \alpha^{b}=
$$

$-f_{c b}^{a}\left(\partial^{v} \partial_{v} \alpha^{c}\right) \alpha^{b}=-f_{b c}^{a}\left(\partial^{v} \partial_{v} \alpha^{b}\right) \alpha^{c}$,

so it gives

$k \frac{\delta \mathcal{L}_{\alpha}}{g}=f_{b c}^{a}\left(\partial_{\nu} \alpha^{b}\right)\left(\partial^{v} \alpha^{c}\right)+f_{b c}^{a}\left(\partial^{v} \partial_{\nu} \alpha^{b}\right) \alpha^{c}$.

From $\delta \mathcal{L}_{\alpha}=0$, one gets

$$
\partial^{v}\left(\left(\partial_{v} \alpha^{b}\right) \alpha^{c}\right)=0
$$

Based on equation (36), $\mathcal{L}_{\alpha}$ is generally not invariant. This shows that the relations between $\alpha^{b}$ and $\alpha^{c}$ are not commutative.

On the other hand for the Grassmann odd with anticommutation relations $\left\{\alpha^{b}, \alpha^{c}\right\}=0$, the $f_{b c}^{a}\left(\partial^{v} \alpha^{b}\right)\left(\partial_{v} \alpha^{c}\right)$ and $f_{b c}^{a}\left(\partial_{v} \alpha^{b}\right)\left(\partial^{v} \alpha^{c}\right)$ parts will satisfy

$$
f_{b c}^{a}\left(\partial^{v} \alpha^{b}\right)\left(\partial_{v} \alpha^{c}\right)=-f_{b c}^{a}\left(\partial_{\nu} \alpha^{c}\right)\left(\partial^{v} \alpha^{b}\right)=
$$

$f_{c b}^{a}\left(\partial_{v} \alpha^{c}\right)\left(\partial^{v} \alpha^{b}\right)=f_{b c}^{a}\left(\partial_{v} \alpha^{b}\right)\left(\partial^{v} \alpha^{c}\right)$

Likewise, the $f_{b c}^{a} \alpha^{b}\left(\partial^{v} \partial_{v} \alpha^{c}\right)$ and $f_{b c}^{a}\left(\partial^{v} \partial_{\nu} \alpha^{b}\right) \alpha^{c}$ parts will satisfy

$$
f_{b c}^{a} \alpha^{b}\left(\partial^{v} \partial_{v} \alpha^{c}\right)=-f_{b c}^{a}\left(\partial^{v} \partial_{v} \alpha^{c}\right) \alpha^{b}=
$$

$f_{c b}^{a}\left(\partial^{v} \partial_{v} \alpha^{c}\right) \alpha^{b}=f_{b c}^{a}\left(\partial^{v} \partial_{v} \alpha^{b}\right) \alpha^{c}$,

so that

$$
k \frac{\delta \mathcal{L}_{\alpha}}{g}=0 .
$$

Based on equation (39) it is clear that the $\alpha^{b}$ parameter fields satisfy Grassmann odd algebra.

The gauge transformation with Grassmann odd parameters is called the BRST transformation. The properties of $\alpha^{a}$ will make $\mathcal{L}_{\alpha}$ invariant so that its addition to the total Lagrangian is valid and provides equations of motion that satisfy the conditions in equation (22) so that $\mathcal{L}_{G F}$ is also invariant. Because the $\alpha^{a}$ field does not appear in experiment, the $\alpha^{a}$ field (or which corresponds to the $\alpha^{a}$ field) is called the ghost field. For example, taking $c^{a}\left(x^{\mu}\right)=-\frac{1}{g} \alpha^{a}\left(x^{\mu}\right)$ will make equation 
(22) the ghost field equation of motion with Lagrangian

$$
\mathcal{L}_{g h}=-i \bar{c}^{a}\left(x^{\mu}\right) \partial^{v} D_{v}^{a b} c^{b}\left(x^{\mu}\right),
$$

which is invariant because $\alpha^{a}$ and $c^{a}$ have the same properties. Therefore an effective Yang-Mills Lagrangian is given by equation (13).

\section{BRST SYMMETRY}

\section{BRST Transformation}

Here we will apply the BRST (BecchiRouet-Stora-Tyutin) transformations to the gauge field (Yang-Mills field) and matter field $\psi$. The gauge transformation for the potential field $A_{\mu}^{a}$ is given by equations (1) and (2), while for the matter field $\psi$ is given by $[1,2]$

$$
\begin{aligned}
& \delta \psi=-i t_{a} \alpha^{a}\left(x^{\mu}\right) \psi \\
& \delta\left(\partial_{\mu} \psi\right)=-i \partial_{\mu}\left(t_{a} \alpha^{a}\left(x^{\mu}\right) \psi\right)
\end{aligned}
$$

The BRST transformation is based on gauge transformation with Grassmann odd transformation parameters [5, 7], as

$$
\begin{aligned}
& \delta_{B} \psi=-i g t_{a} \eta^{a}\left(x^{\mu}\right) \psi \\
& \delta_{B}\left(\partial_{\mu} \psi\right)=-i g \partial_{\mu}\left(t_{a} \eta^{a}\left(x^{\mu}\right) \psi\right) \\
& \delta_{B}\left(A_{\mu}\right)=-t_{a} D_{\mu}^{a b} \eta^{b}\left(x^{\mu}\right) \\
& \delta_{B}\left(A_{\mu}^{a}\right)=-D_{\mu}^{a b} \eta^{b}\left(x^{\mu}\right)
\end{aligned}
$$

with a mathematical relation, for example, $\eta^{a}\left(x^{\mu}\right)=-c^{a}\left(x^{\mu}\right)=\frac{1}{g} \alpha^{a}\left(x^{\mu}\right)$. This relation is not unique, provided that the gauge fixing Lagrangian invariant conditions are fulfilled, in the case of the Lorenz gauge given by equation (22). Any Lagrangian that is invariant to the gauge transformation will also be BRST invariant. In the BRST transformation, the properties of the transformation parameters are also extended by making the BRST transformation parameters transformed as $\delta_{B}\left(\eta^{a}\left(x^{\mu}\right)\right) \neq 0$ [5].

Based on the Grassmann odd properties of the parameters $\eta^{a}\left(x^{\mu}\right)$ and the Lie algebra of the BRST transformation generator, the BRST transformation generators satisfy $t_{a} t_{b}=-t_{b} t_{a}$. The BRST transformation requires $\delta_{B} \delta_{B} \equiv 0$, known as the nilpotent property [5]. Because of this nilpotent property, $\delta_{B}$ acts as a Grassmann odd object. This has the advantage that any Lagrangian with a BRST variation form will have an invariant BRST action. From the nilpotent properties, $\delta_{B}\left(\delta_{B} \psi\right)$ and $\delta_{B}\left(\delta_{B}\left(A_{\mu}^{a}\right)\right)$, we can produce the expression for the transformation parameter $\eta^{a}\left(x^{\mu}\right)$ in the form of BRST variation.

For $\delta_{B}\left(\delta_{B} \psi\right)$ it will give
$\delta_{B}\left(\delta_{B} \psi\right)=-i g t_{a}\left(\delta_{B}\left(\eta^{a}\right)-\frac{1}{2} g f_{b c}^{a} \eta^{b} \eta^{c}\right) \psi$

and for gauge field, it will give

$\delta_{B}\left(\delta_{B}\left(A_{\mu}^{a}\right)\right)=D_{\mu}^{a j}\left(-\delta_{B}\left(\eta^{j}\left(x^{\mu}\right)\right)+\right.$

$\left.\frac{1}{2} g\left(f_{b c}^{j} \eta^{b}\left(x^{\mu}\right) \eta^{c}\left(x^{\mu}\right)\right)\right)$.

From these two results $\delta_{B} \delta_{B}$ will only vanish for all fields if

$$
\delta_{B}\left(\eta^{a}\left(x^{\mu}\right)\right)=\frac{1}{2} g f_{b c}^{a} \eta^{b}\left(x^{\mu}\right) \eta^{c}\left(x^{\mu}\right)
$$

Because the ghost field $c^{a}\left(x^{\mu}\right)=-\eta^{a}\left(x^{\mu}\right)$ has the same properties (Grassmann Odd) then

$$
\delta_{B}\left(c^{a}\left(x^{\mu}\right)\right)=-\frac{1}{2} g f_{b c}^{a} c^{b}\left(x^{\mu}\right) c^{c}\left(x^{\mu}\right)
$$

\section{Effective BRST Lagrangian}

Having obtained expression of BRST transformation parameters $\eta^{a}\left(x^{\mu}\right)$ and ghost field $c^{a}\left(x^{\mu}\right)$ in $\delta_{B}\left(\eta^{a}\left(x^{\mu}\right)\right)$ and $\delta_{B}\left(c^{a}\left(x^{\mu}\right)\right)$, now, the ghost field and the anti-ghost field are treated as an independent field. Define

$$
\delta_{B}\left(\bar{c}^{a}\left(x^{\mu}\right)\right)=B^{a}\left(x^{\mu}\right)
$$

where $B^{a}\left(x^{\mu}\right)$ is a scalar field, LautrupNakanishi auxiliary field [6, 7]. Using the nilpotent property of the BRST transformation

$$
\delta_{B}\left(B^{a}\left(x^{\mu}\right)\right)=\delta_{B}\left(\delta_{B}\left(\bar{c}^{a}\left(x^{\mu}\right)\right)\right)=0 .
$$

That is, it can be added to any Lagrangian term which is a variation of BRST [6], so that

$$
\mathcal{L}=\mathcal{L}_{Y M}+\delta_{B} \mathcal{O} .
$$

Here $\mathcal{L}_{Y M}$ is BRST invariant because it is gauge invariant. The Lagrangian term $\delta_{B} \mathcal{O}$ corresponds to the fixing of the gauge on the Yang-Mills field. Choose

$\mathcal{O}\left(x^{\mu}\right)=\bar{c}^{a}\left(x^{\mu}\right)\left[-\frac{1}{2} \xi B^{a}\left(x^{\mu}\right)+i G^{a}\left(x^{\mu}\right)\right]$

where $G^{a}\left(x^{\mu}\right)$ is the gauge fixing term [6]. For example, using Lorenz gauge $G^{a}\left(x^{\mu}\right)=\partial^{\mu} A_{\mu}^{a}$, then

$$
\delta_{B} \mathcal{O}\left(x^{\mu}\right)=\left(\delta_{B} \bar{c}^{a}\right)\left[-\frac{1}{2} \xi B^{a}\left(x^{\mu}\right)+\right.
$$

$\left.i \partial^{\mu} A_{\mu}^{a}\right]-\bar{c}^{a}\left(x^{\mu}\right)\left[-\frac{1}{2} \xi\left(\delta_{B} B^{a}\left(x^{\mu}\right)\right)+\right.$

$\left.i \partial^{\mu}\left(\delta_{B} A_{\mu}^{a}\right)\right]$.

Therefore,

$$
\begin{aligned}
& \delta_{B} \mathcal{O}\left(x^{\mu}\right)=-\frac{1}{2} \xi B^{a} B^{a}+i B^{a} \partial^{\mu} A_{\mu}^{a}- \\
& i \bar{c}^{a}\left(x^{\mu}\right) \partial^{\mu}\left(D_{\mu}^{a b} c^{b}\left(x^{\mu}\right)\right) .
\end{aligned}
$$

This additional Lagrangian term will give the equation of motion for the field $B^{a}$ i.e.

$$
\frac{\partial\left(\delta_{B} \mathcal{O}\left(x^{\mu}\right)\right)}{\partial\left(B^{a}\left(x^{\mu}\right)\right)}=\xi B^{a}\left(x^{\mu}\right)-i \partial^{\mu} A_{\mu}^{a}=0
$$

By substituting eq. (57) in eq. (56) it is obtained 


$$
\begin{aligned}
& \delta_{B} \mathcal{O}\left(x^{\mu}\right)=-\frac{1}{2 \xi}\left(\partial^{\mu} A_{\mu}^{a}\right)\left(\partial_{v} A_{a}^{v}\right)- \\
& i \bar{c}^{a}\left(x^{\mu}\right) \partial^{\mu}\left(D_{\mu}^{a b} c^{b}\left(x^{\mu}\right)\right)
\end{aligned}
$$

With the gauge fixing Lagrangian is the same as equation (20) and the ghost field Lagrangian is the same as equation (40). Thus, the effective Lagrangian which is BRST invariant for the YangMills field is the Lagrangian that has been shown in equation (13).

In addition, through the Faddeev-Popov method on the Yang-Mills and by gauge fixing the Lagrangian by inserting the BRST invariant identity

$$
1=\Delta_{\mathrm{F}}\left(A_{\mu}^{U}\right) \int D \eta \delta\left(F^{a}\left[A_{\mu}^{U}\right]\right)=
$$

$\Delta_{\mathrm{F}}\left(A_{\mu}\right) \int D \eta \delta\left(F^{a}\left[A_{\mu}\right]\right)$

a propagator with the same form in equation (10) will be obtained with the effective Lagrangian given by equation (11). The matrix $M_{a b}$ will be as in equation (12) so that the effective Lagrangian will turn into equation (13).

\section{CONCLUSION}

The least action principle gives the Grassmann odd properties of the ghost fields and their relations to the gauge transformation and BRST transformation parameters. This relations is not unique but must make the effective Lagrangian invariant conditions fulfilled. It has also been proven that by applying the Faddeev-Popov method to the Lagrangian Yang-Mills in BRST symmetry, the ghost field expression is obtained.

The least action principle, BRST variations and path integrals provide the same effective Lagrangian. This shows that the least action principle method is valid to be used in finding relations between ghost fields and transformation parameters. Also in BRST symmetry, path integral quantization of the gauge field with constraints will provide the same propagator as the effective propagator in gauge symmetry. This shows that the effective Lagrangian is BRST invariant and fulfills the path integral quantization. For further research, we need to explore the canonical quantization of the effective Lagrangian, BRST symmetry and its relations to the Noether current, and BRST Hamiltonian formalism.

\section{REFERENCES}

[1] Peskin, M. E., \& Schroeder, D. V. An Introduction to Quantum Field Theory. Reading, Massachusetts: Perseus Books Publishing LLC. (1995).

[2] Ryder, L. H. Quantum Field Theory. Cambridge: Cambridge University Press. (1996).

[3] Faddeev, L. D., \& Popov, V. N. Feynman Diagrams for the Yang-Mills Field. Physics Letter B, Vol. 25, 29-30. (1967). DOI: https://doi.org/10.1016/0370-2693(67)90067-6

[4] Berezin, F. A. The Method of Second Quantization. Orlando, Florida: Academic Press Inc. (1966).

[5] Becchi, C., Rouet, A., \& Stora, R. Renormalization of Gauge Theories. Annals of Physics. 98, 287-321. (1976). DOI: https://doi.org/10.1016/0003-4916(76)90156-1

[6] Srednicki, M. Quantum Field Theory. Santa Barbara, California: University of California, Santa Barbara. (2006).

[7] Kugo, T., \& Ojima, I. Local Covariant Operator Formalism of Non-Abelian Gauge Theories and Quark Confinement Problem. Supplement of the Progress of Theoretical Physics, No. 66. (1979). DOI: https://doi.org/10.1143/PTPS.66.1 